\title{
BMJ Open Estimation of minimally important difference of the EQ-5D-5L utility scores among patients with either hypertension or diabetes or both: a cross-sectional study in Hong Kong
}

\author{
Richard Huan XU (D) , Eliza Lai-yi WONG (D) , Annie Wai-ling CHEUNG
}

To cite: $X U$ RH, WONG EL, CHEUNG AW. Estimation of minimally important difference of the EQ-5D-5L utility scores among patients with either hypertension or diabetes or both: a cross-sectional study in Hong Kong. BMJ Open 2020;10:e039397. doi:10.1136/ bmjopen-2020-039397

- Prepublication history for this paper is available online. To view these files, please visit the journal online (http://dx.doi. org/10.1136/bmjopen-2020039397).

Received 14 April 2020 Revised 03 November 2020 Accepted 11 November 2020

D) Check for updates

(c) Author(s) (or their employer(s)) 2020. Re-use permitted under CC BY-NC. No commercial re-use. See rights and permissions. Published by BMJ.

Centre for Health Systems and Policy Research, Jockey Club School of Public Health and Primary Care, The Chinese University of Hong Kong, Hong Kong SAR, China

Correspondence to Dr Richard Huan XU; richardhxu@cuhk.edu.hk and Professor Eliza Lai-yi WONG; lywong@cuhk.edu.hk

\section{ABSTRACT}

Objective To estimate and report the minimally important difference (MID) of the EuroQol five-dimensional five-level questionnaire (EQ-5D-5L) using the Hong Kong (HK) utility score among patients with either hypertension or diabetes or both.

Design Data were analysed using secondary data analysis based on a cross-sectional study assessing patients' experiences in HK.

Setting A representative sample was recruited from 26 specialist outpatient clinics in HK.

Participants We analysed data from 2231 and 662 patients who reported having hypertension or diabetes alone, respectively, and 874 patients had these two diseases.

Intervention An instrument-defined approach was applied to estimate MID stratified according to sex and age for the three subpopulations.

Results The overall MID (OMID) estimates were 0.089 , 0.086 and 0.089 for patients with hypertension or diabetes alone and with these two diseases, respectively. The adjusted MID (aMID) estimate was smaller than the oMID, and the improved MID was larger than the deteriorated MID. Women had larger oMID but smaller aMID than men. Younger respondents had larger aMID than older respondents. Effect sizes ranged from 0.30 to 0.503 , which fit our preset criteria.

Conclusion Four types of MID of the EQ-5D utility score for patients with hypertension or diabetes alone and with these two diseases were reported. Variations in the MID estimates should be further explored in other populations or using different statistical methods.

\section{INTRODUCTION}

Hypertension is common among patients with diabetes, with the prevalence depending on the type and duration of diabetes, as well as other demographic characteristics, such as sex and age. ${ }^{1}$ Patients with hypertension have twice the risk of having diabetes than those without hypertension. ${ }^{2}$ Studies indicated a pathogenic association between diabetes and hypertension, that is, patients with
Strength and limitations of this study

- An array of values representing minimally important changes was provided for the EuroQol fivedimensional five-level questionnaire utility score.

- Four types of minimally important difference (MID) stratified according to sex and age were reported for patients with either hypertension or diabetes or both.

- The instrument-defined approach was first applied to estimate the MIDs for patients with both hypertension and diabetes.

- The MID values estimated in this study should be further examined using the other methods.

hypertension often have insulin resistance, which poses a great risk for the development of diabetes. Conversely, a major cause of morbidity and mortality in patients with diabetes is caused by cardiovascular diseases, which are triggered by hypertension. ${ }^{3}$ It is predicted that the number of people with diabetes will reach 642 million by $2040,{ }^{4}$ and the prevalence of hypertension is more common, with an estimate of 1.39 billion worldwide. ${ }^{5}$ As a chronic condition, either hypertension or diabetes is negatively associated with physical and psychological healthrelated quality of life (HRQoL), which is an important outcome measurement in healthcare. ${ }^{6}$ Recently, the HRQoL of patients with hypertension or diabetes has been reported worldwide using patient-reported outcome measures (PROMs) ${ }^{7-9}$ However, the marginal variations in the HRQoL that lead to substantial changes in patient well-being cannot be directly measured, especially when patients have multimorbidity of chronic conditions.

To examine the responsiveness of changes in the health status and HRQoL in clinical practice or primary healthcare, there have 
been a number of studies defining and calculating the minimally important difference (MID), an important means of interpreting changes in the difference in HRQoL. ${ }^{10}$ MID is defined as a measure to indicate the smallest change in the utility using the PROMs of interest, either beneficial or harmful, and that change represents a meaningful transformation for the patient's health states and can help medical professionals comprehensively evaluate the efficiency of clinical interventions. ${ }^{112}$ This kind of MID estimation uses a patient-centred approach and explores not only the meaningful physical or biological changes to patients but also the value that patients attribute to changes according to their HRQoL. ${ }^{13}$ Although the estimation of MID of the utility score determined using PROMs has been reported frequently, ${ }^{14-16}$ MID estimates focused on specific conditions are limited, especially for comorbidities, such as hypertension and diabetes. In addition, MID estimates are likely to vary in different populations by considering the local preferences. ${ }^{12}$ It is necessary to have country/region-specific estimation for clinical or health economics evaluation analysis.

The EuroQol five-dimensional questionnaire (EQ-5D) is among the most widely used generic preference-based measures (GPBMs) for measuring HRQoL worldwide. ${ }^{17}$ It is recommended as the standard measure in the estimation of quality-adjusted life years (QALYs) used for the economic evaluation in most European countries. ${ }^{18-20}$ Currently, in addition to clinical interventions, the EQ-5D is increasingly used in facilitating decisionmaking in primary care settings to manage patients with chronic conditions; however, no study has estimated the MID for patients with hypertension or diabetes alone and with both the diseases by applying local EuroQol fivedimensional five-level questionnaire (EQ-5D-5L) utility scores in Hong Kong (HK). Therefore, this study aimed to estimate and report the MID estimate of the EQ-5D-5L utility score among patients with either hypertension or diabetes or both using a sample from HK.

\section{METHOD}

\section{Data source}

Data used for the estimation of MID in this study were derived from a regional-wide annual patient experience survey of individuals using public healthcare services in HK. $^{21}$ The patients who visited any of the 26 selected public specialist outpatient clinics located in various regions of HK were randomly invited to participate in the survey during the study period. The inclusion criteria were age $\geq 18$ years and being able to speak and understand Cantonese. Patients who were day cases or day surgeries or those from paediatric, hospice, psychiatric, dental, anesthesiology, pathology or nurse-led, or multispecialty outpatient clinics were excluded. The responding patients who agreed to participate in the telephone interview were asked to report their experience of service used using a structured and validated questionnaire. ${ }^{22}$ The questions about self-reported HRQoL (using
EQ-5D-5L HK) and long-standing health conditions were also included. A total of 13966 patients responded to the survey and provided valid responses. Among them, a total of 2231 and 662 patients reported having hypertension and diabetes alone, respectively, and 874 patients reported having both conditions. Data from all subjects were used for the estimation of MID in this study.

\section{HRQoL measurement}

The EQ-5D-5L is the advanced version of the previous EQ-5D-3L that can better control the ceiling effect than the EQ-5D-3L. ${ }^{23}$ It has five dimensions: mobility (MO), self-care, usual activities, pain/discomfort and anxiety/ depression. Each dimension has five levels (no, slight, moderate, severe and unable/extreme problems). The combinations of the dimensions and levels could generate a total of $3125\left(5^{5}\right)$ health states. The health states can then be converted into a summary utility score based on local value set and facilitate the generation of QALYs for health economic evaluation. ${ }^{24}$ The available EQ-5D value sets for different countries/regions are listed in the EuroQoL's website (euroqol.org/publications/keyeuroqol-references/value-sets/). In the present study, the EQ-5D-5L utility score was derived based on the HK population tariff. ${ }^{25}$ The mean utility value of the HK value set was 0.919 . The normative profile of the HK general population was reported by Wong et al. ${ }^{26}$

\section{Statistical analyses}

Descriptive statistics was used to describe respondents' demographic characteristics. The EQ-5D-5L utility score was reported in means and SD and stratified according to health conditions (with hypertension or diabetes alone, and with both hypertension and diabetes). $\mathrm{R}$ (R Foundation, Austria) was used for data analyses.

The instrument-defined approach was adopted to estimate the MIDs of the HK EQ-5D-5L utility score. ${ }^{16}$ This method is a variant of the anchor-based method in which the internal anchors, rather than the external anchors, are used to estimate the MID. ${ }^{16}$ In this study, the MIDs were estimated for patients with hypertension and diabetes alone and with both diseases. To explore the heterogeneity of the MID estimates attributed to patient characteristics, we further stratified the estimation according to sex (male and female) and age ( $\leq 55$ years, $56-65$ years, $66-75$ years and $\geq 76$ years) for each subpopulation.

The MID was estimated based on the average scores of differences between the baseline health states and single-level transitions to the other adjacent health states; for example, the baseline health state is '22222' (slight problem on all five dimensions), a possible adjacent health state of single-level transition toward worse health state could be '32222' (moderate problem on MO and slight problem on the other dimensions), and another possible adjacent health state of single-level transition toward better health state could be '12222' (no problem on MO and slight problem on the other dimensions). A single MID estimate of the baseline health state was derived 


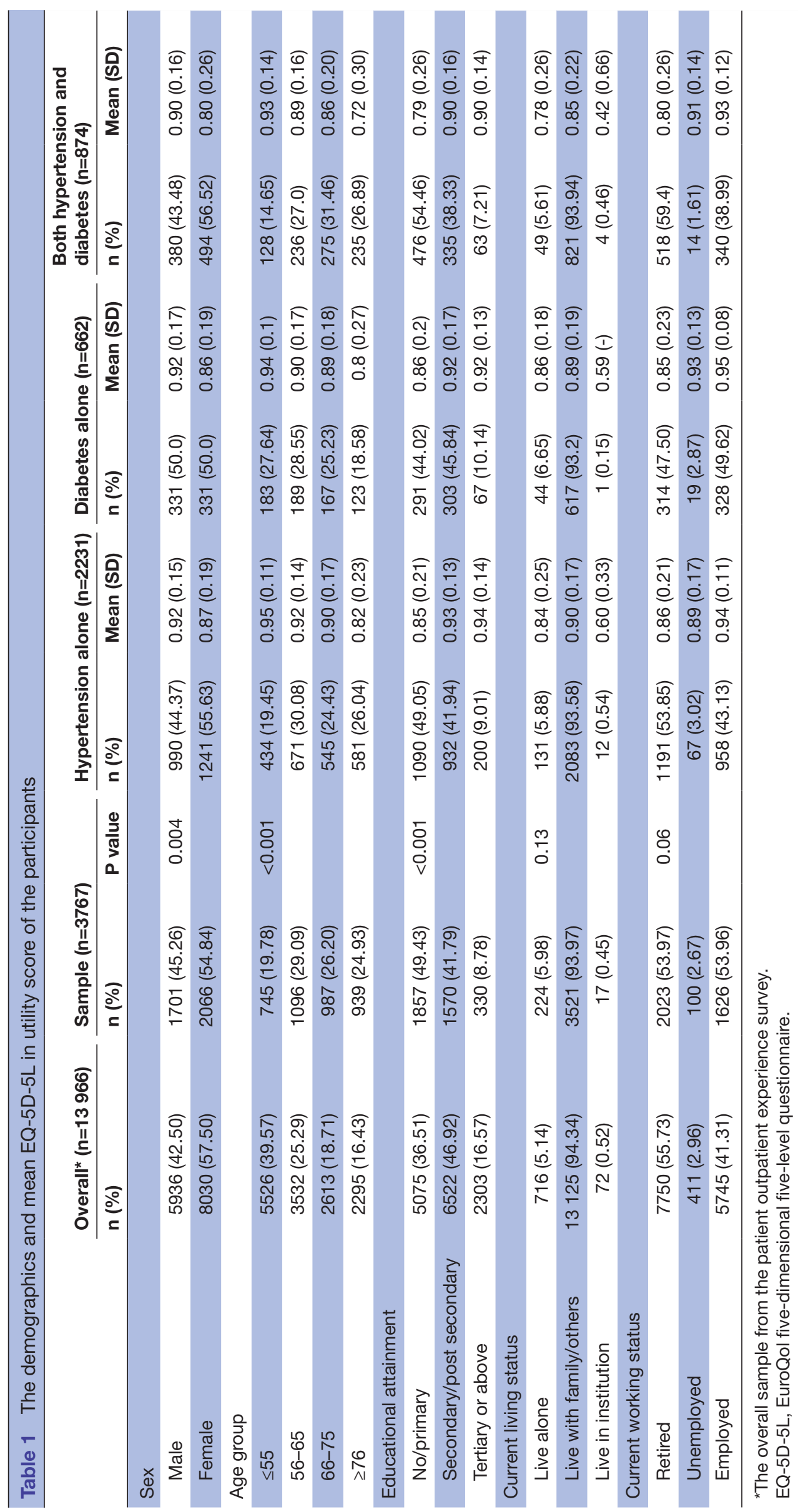




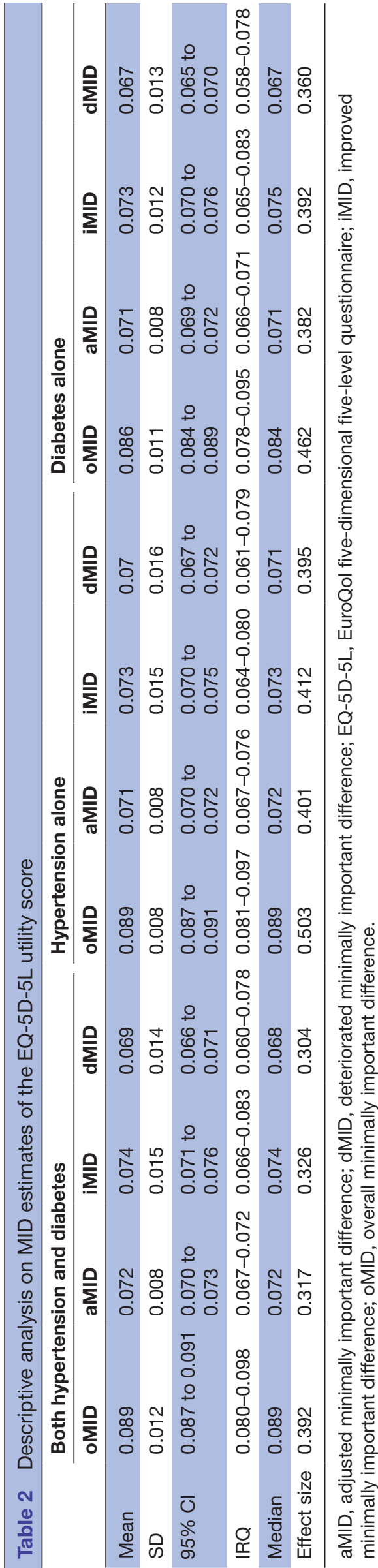

by averaging the sum of the absolute utility score of all possible adjacent health states. Details of the instrumentbased method are found elsewhere. ${ }^{12} 16$ Apart from the overall MID (oMID) reported in our study, two types of MID were presented as well: one was the improved MID (iMID), which was the health state changed toward the better direction, for example, from '22222' to '12222'; the other one was the deteriorated MID (dMID), which was the health state changed toward the worse direction, for example, from '22222' to '32222'. ${ }^{14}$ However, we noticed that the health states ' 11111 ' and ' 55555 ' were two extremist health states, which indicate that no health state could be better than the best state ('11111') or worse than the worst state (' 55555 '). Thus, no iMID for ' 11111 ' and no dMID for ' 55555 ' were estimated. Moreover, a previous study suggested that to avoid a larger single-level transitions in EQ-5D than in the MID estimate, the maximum value of the single-transition among different levels of dimensions should be excluded from the MID estimate. ${ }^{12}$ Reviewing the HK scoring algorithm, the differences between level 3 (moderate problem) and level 4 (severe problem) were larger than those among the other levels $(0.189,0.158,0.14,0.16$ and 0.153$){ }^{25}$ Thus, the other type of MID, adjusted MID (aMID), was reported based on excluding the maximum value scoring parameters. Overall, four types of MID that were stratified according to age and sex were reported for three subpopulations. The effect size (ES) was calculated to indicate whether the magnitude of the MID estimate is larger than the measurement error or the minimally detectable difference of the EQ-5D ${ }^{27}$ The plausible range of ES was $0.2-0.8$, ES $<0.2$ indicating that the MID estimate was unlikely to be 'important' and ES $>0.8$ indicating that the MID estimate was unlikely to be 'minimal'. ${ }^{28}$ The locally weighted scatterplot smoothing (LOESS) smoothing approach was used to estimate the association between the observed utility score and MID estimates. ${ }^{29}$ Considering that only $4.08 \%, 4.83 \%$ and $6.18 \%$ of the respondents with hypertension, diabetes and both, respectively, reported utility scores $<0.5$, only the association in MID estimates with a utility scores $\geq 0.5$ were investigated.

\section{Patient and public involvement}

No patients were involved in the design of the study.

\section{RESULTS}

Table 1 shows that women constitute $56.5 \%$ of the respondents with both hypertension and diabetes. Respondents with diabetes were younger than those with hypertension. Nearly half of the respondents reported completing primary education or less. More than $90 \%$ of respondents were living with their families, and very few were unemployed $(1.6 \%-3.0 \%)$. The mean EQ-5D utility values for each demographic group are presented in table 1 . Compared with the profile of overall sample from the original patient experience survey, more male and older 


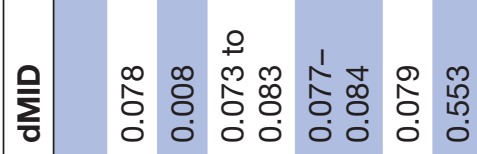

ำํำ $\infty$

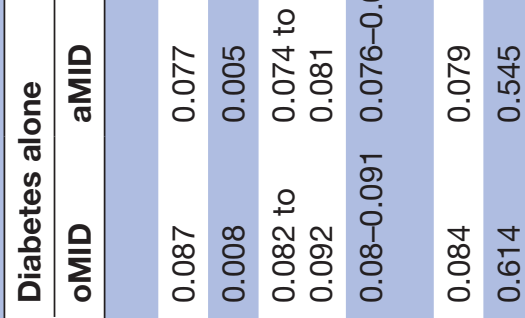

产

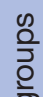

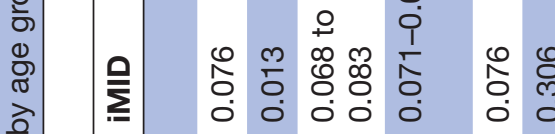

क्ष

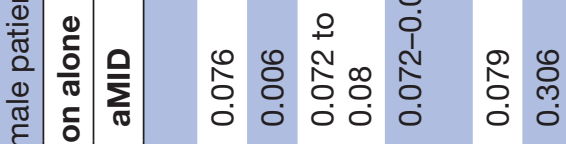

항

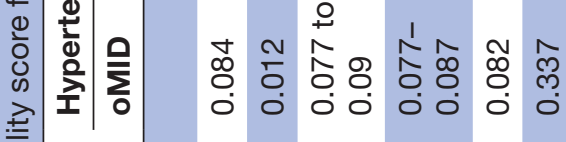

पे

常

产

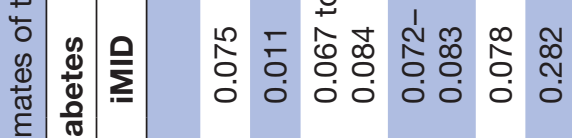

㰻

政

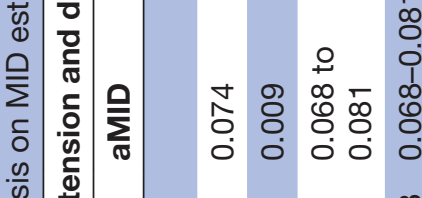

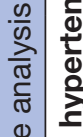

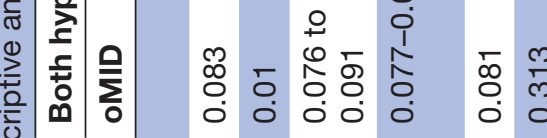

远

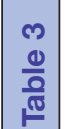

र

o o o o o o o

ก.

० 0 0 0 0.

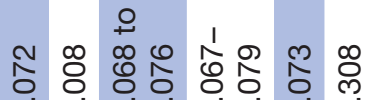

o o o o o o o

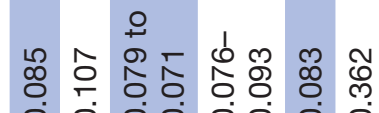

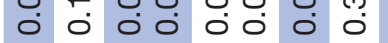

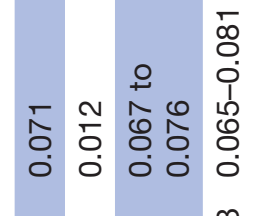

$\infty$

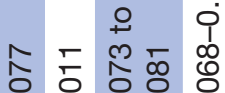

o.

$\begin{array}{ll}0 & \infty \\ 0 & \stackrel{m}{m} \\ 0 & 0\end{array}$

ㅇํㅇ

००

잉

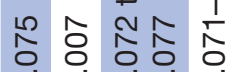

동 응

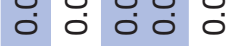

○ं

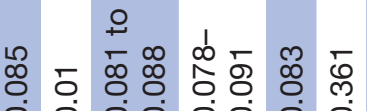

o o O O O O O O

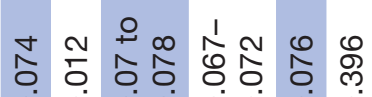

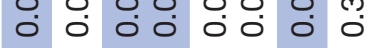

ำ

o o 00000

$\stackrel{0}{\circ}$

4

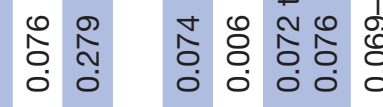

$\begin{array}{lllll}0 & 0 \\ 0 & 0 & 0 \\ 0 & 0 & 0 & 0 \\ 0 & 0 & 0 & 0 \\ 0 & 0 & 0 \\ 0\end{array}$

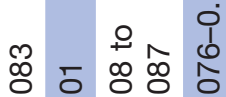

O. 잉ㅇㅇㅇ.

๓

ㅇํㅇ

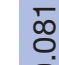

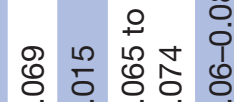

○ ०ᄋㅇㅇㅇ

กิ

0.0

0

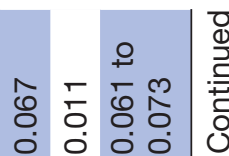

m

○

웅

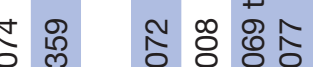

$\circ 0^{\circ}$

(n)

$\vec{\omega}$

응.

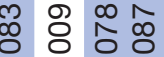

○ 0 0ं

윽 


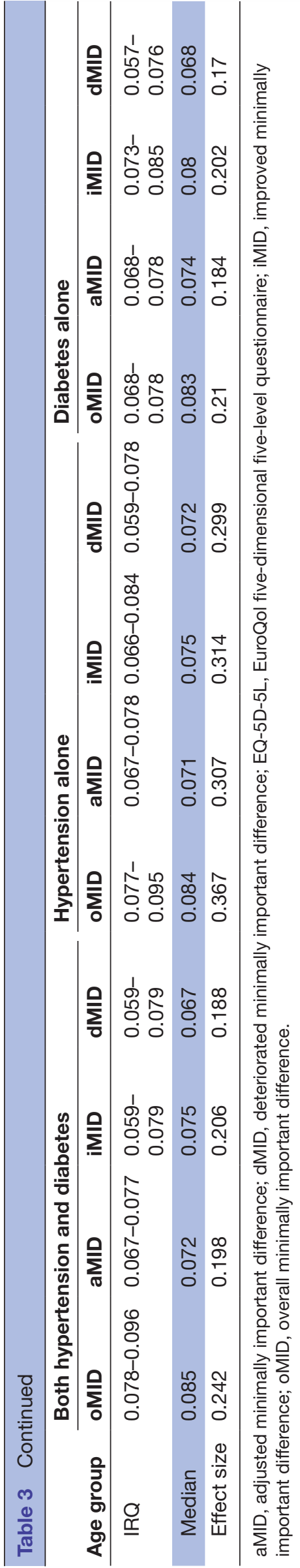

people with lower educational levels were included in our study.

Tables 2-4 present the MID estimates for all respondents, which were stratified according to sex and age. The oMID estimates were $0.089,0.086$ and 0.089 for patients with hypertension or diabetes alone and with these two diseases, respectively. The aMID estimate was smaller than the oMID estimate, and the iMID estimate was larger than the dMID estimate. The SD, 95\% CI, IQR and median of all the MID estimates were reported separately in the tables. The maximum discrepancies of the MID estimates between hypertension and diabetes were 0.003 on aMID for men (56-65 years) and women (56-65 years and 75 years). Additionally, young respondents demonstrated larger aMID but smaller oMID than old respondents. Difference in the MID estimates among different age groups was smaller for patients with both hypertension and diabetes than for those with either of them alone. The ES ranged from 0.17 to 0.636 , which best fits our preset criteria. Figure 1 shows the positive association between aMID estimates and the observed utility scores, with the MID estimates increasing with increasing utility scores.

\section{DISCUSSION}

MID is a standard approach in the interpretation of clinical relevance of changes in HRQoL; the nuance of MID reflects the effectiveness of the clinical interventions in changing patient HRQoL in terms of their characteristics and health status. This study reported four types of MID estimates of the EQ-5D-5L HK utility score that were stratified according to sex and age of patients with hypertension or diabetes alone or with both conditions. Overall, patients with both hypertension and diabetes reported larger aMIDs and iMIDs than those with hypertension or diabetes alone. For dMID, patients with diabetes alone reported smaller estimates than those with hypertension alone or with both conditions.

Few studies have estimated the MID either based on the utility score of EQ-5D-5L for specific conditions. Our previous study reported a larger aMID estimate of 0.079 (range: 0.048-0.084) for patients with chronic conditions using a sample from HK. ${ }^{30}$ However, we did not differentiate chronic conditions or identify the number of chronic conditions that a patient may have. McClure et al reported smaller aMID, iMID, and dMID estimates than those of the present study for patients with diabetes and associated multimorbidity using an instrument-defined method in a sample from Canada. ${ }^{14}$ However, they did not report the types of multimorbidity and did not further explore variations in the MID estimates in terms of patient characteristics. Tsiplova $e t$ $a l$ identified a larger oMID of the EQ-5D-5L utility score for patients with hypertension (0.093) or diabetes (0.098) separately using an anchor-based method. ${ }^{31}$ Despite the fact that the instrument-defined approach should be encouraged because of its simple operation and estimation based on multianchors, the MID estimates rely heavily on the scoring 


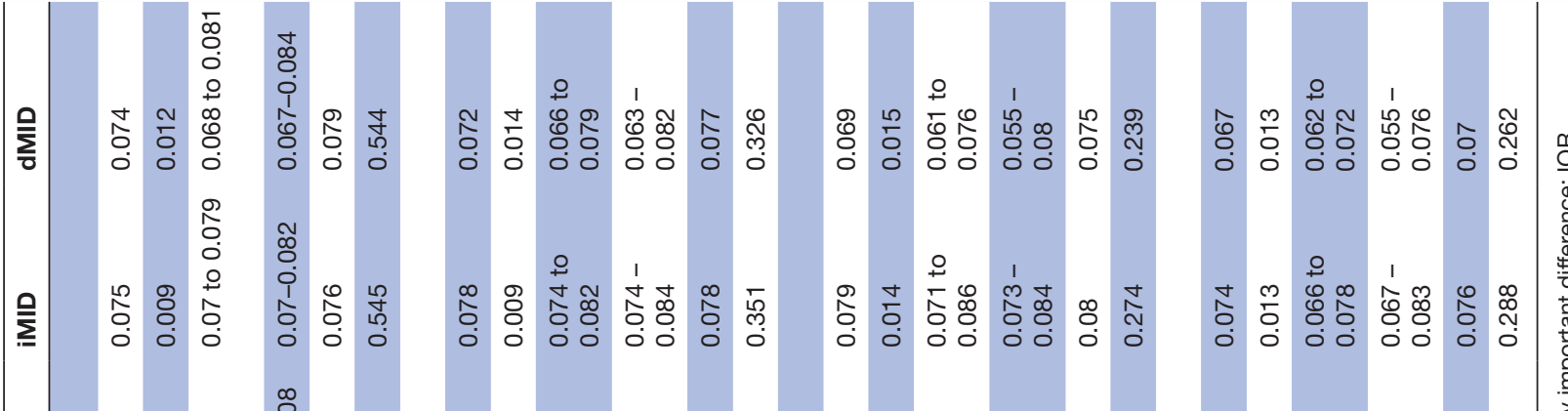

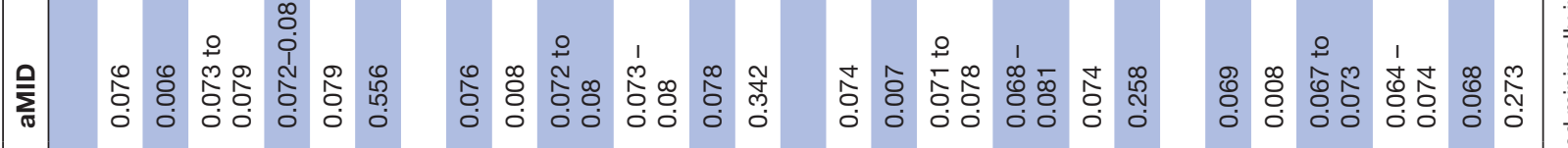

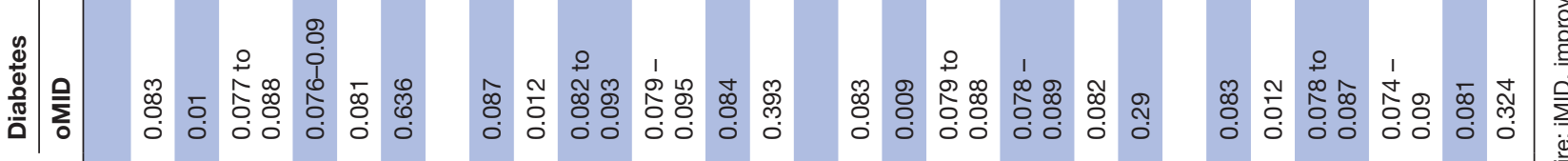

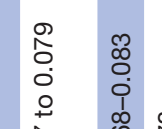

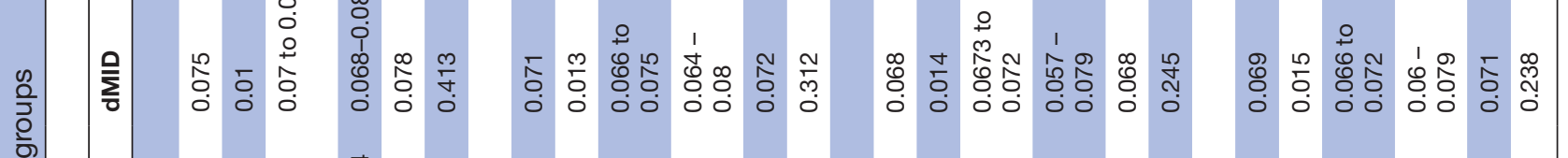

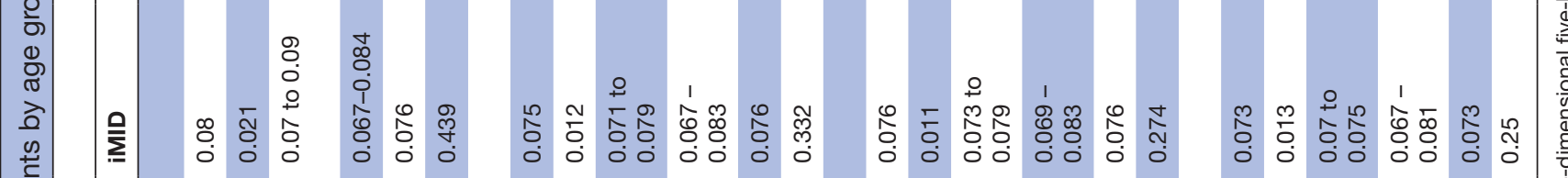

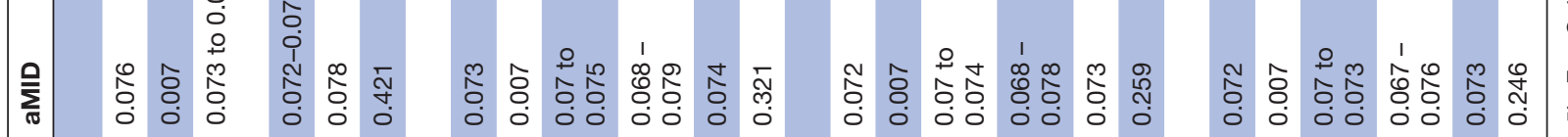

\section{o.}

每

$\overbrace{0}^{0}$

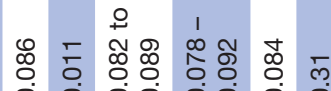

舟

$\approx$

เִ

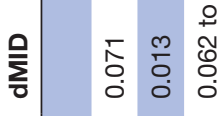

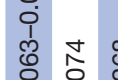

i.

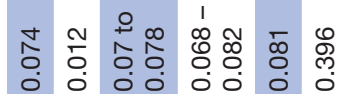

010

0

m

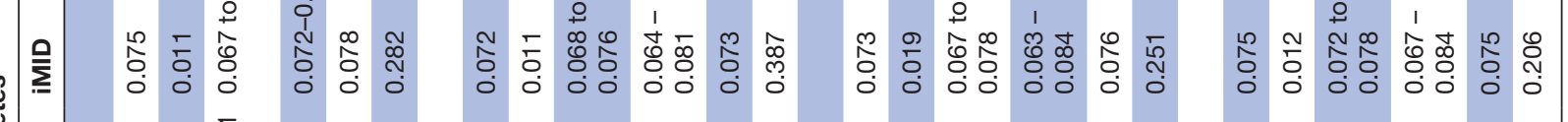
-

0
0
0
0
$\infty$

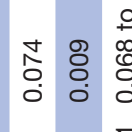

$\begin{array}{lll}0 & 0 \\ 0 & 0 & 0 \\ 0 & 0 & 0 \\ 0 & 0 & 0\end{array}$

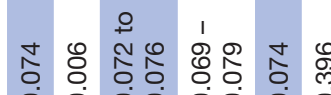

$\circ$ o

O.

$\infty$ ㅇํํ.

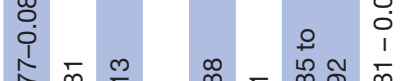

\begin{tabular}{lll}
$\hat{0}$ & $\infty$ & \multicolumn{2}{c}{} \\
0 & 0 & 0 \\
0 & 0 \\
0
\end{tabular}

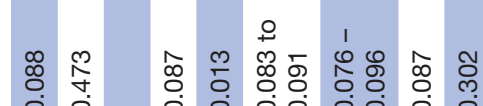

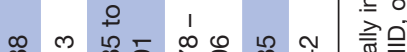
竞

离

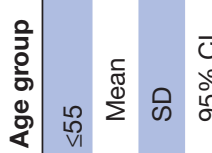

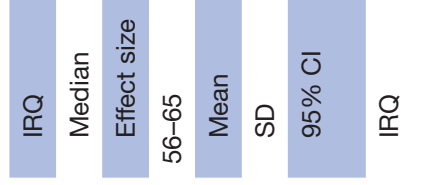

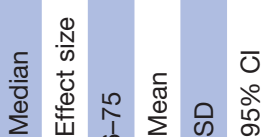

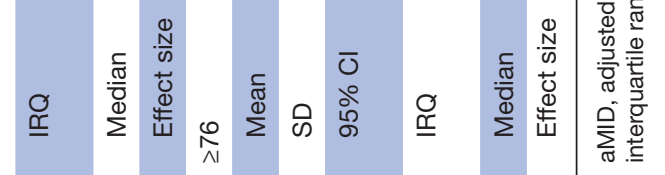



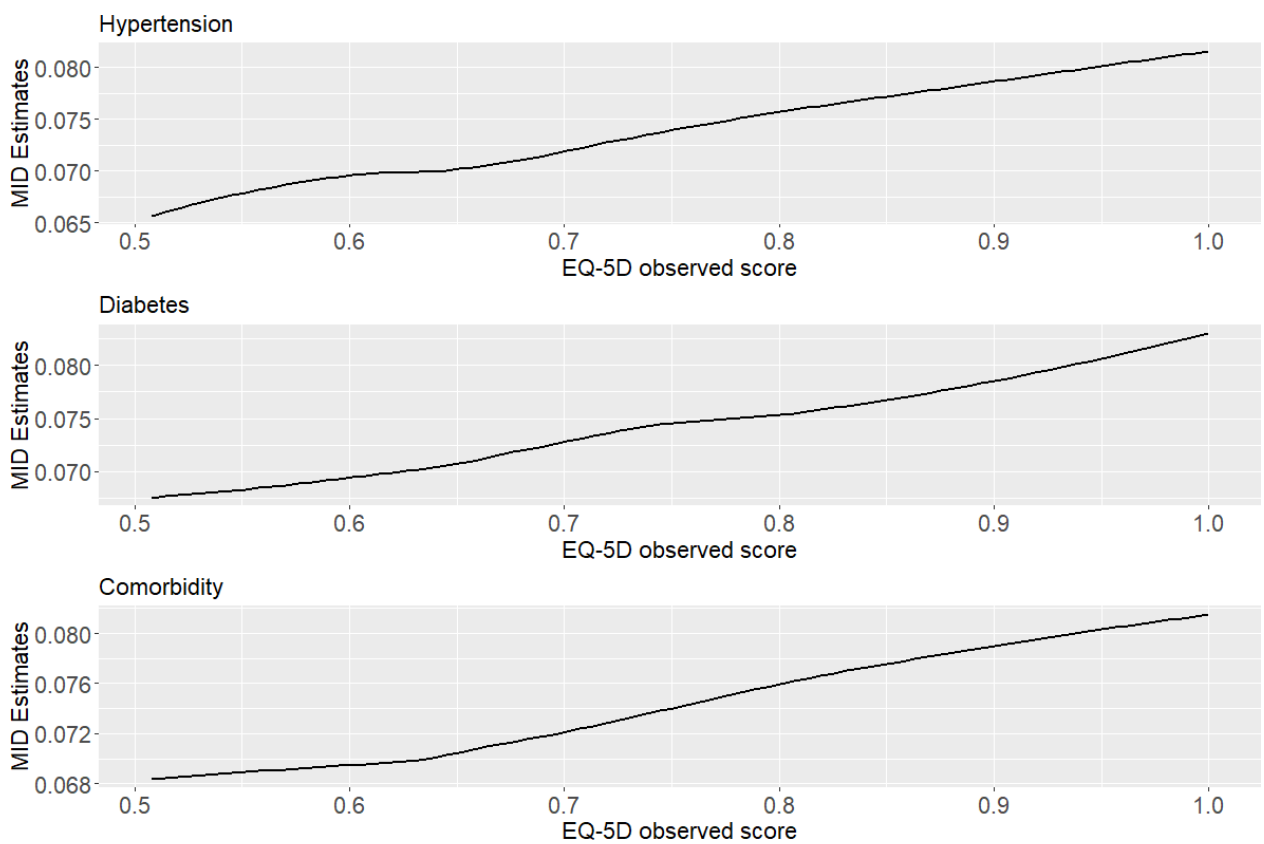

Figure 1 The relationship between the EuroQol five-dimensional five-level questionnaire (EQ-5D-5L) observed score and adjusted minimally important difference (MID) estimates; comorbidity=both hypertension and diabetes.

algorithms adopted. Further studies on the comparisons of MID estimates of the EQ-5D-5L utility value among patients with both hypertension and diabetes using other scoring algorithms are needed.

Given the ageing of the large baby boomer cohort coupled with the advancement of medical technology, which means that a growing number of people might be living with multimorbidity, estimations of the MID in HRQoL for this population become more important. ${ }^{12}{ }^{32}$ In the present study, patients with both hypertension and diabetes demonstrated larger MID estimates than those with hypertension or diabetes alone, suggesting that achieving meaningful changes in patient HRQoL might be difficult through clinical or primary care interventions when both conditions are present. Previous studies also confirmed that there is an urgent need to improve hypertension care and blood pressure control strategies in patients with diabetes. ${ }^{18}$ Additionally, larger estimates for iMID than for dMID suggest that for the same utility score, a smaller change to worse health state should be considered important, whereas the same magnitude of change to a better health state may be less important. However, in McClure et al.'s study, an equal iMID and dMID for patients with diabetes was reported. ${ }^{14}$ For patients with chronic conditions, the outcomes of healthcare should not be evaluated to restore physical health but to improve their HRQoL in order for them to live in independence, dignity and comfort. ${ }^{33}$ The MID estimate in multimorbidity of chronic conditions is essential for providing meaningful interpretation of the effects of an intervention on HRQoL and to classify changes in patient well-being.

The MID estimates varied across the distribution of sex and age. Smaller MID estimates were reported in older adults than in younger respondents in both men and women; the accumulated experience in dealing with health problems in elderly patients may have affected our MID estimates. ${ }^{34}$ However, the heterogeneities of the MID estimates between patient groups with different demographic characteristics are negligible, and all the differences are smaller than 0.01 . This result suggests that in clinical practice, the differences in MID estimates using EQ-5D-5L between male and female or young and old HK patients with chronic conditions may not be important. Nevertheless, our findings regarding MID estimates stratified according to sex and age offer evidence for validating the oMID estimate from another perspective. The range provided a 'CI' of oMID estimate that may help clinical professionals and researchers to better assess the effectiveness of clinical interventions. Further studies are needed to investigate whether the variations are significant and valid in other HK patient groups. Additionally, the severity of conditions, treatment context, and interactions with the socioeconomic status may have an influence on the nuances in the MID estimates ${ }^{35}$ and should be considered in future studies.

MID is an important patient-centred concept to facilitate decision-making on the appropriate intervention that meets patients' preference and values and to provide clinical or political priorities. ${ }^{28}$ For clinical professionals, MID estimates could help them to make decisions from the perspective of patient-centred care to determine treatment options or decisions regarding termination of the treatment for individual patients. On the macro level, MID estimates could be used as thresholds to confirm the effectiveness of a new treatment as opposed to the current best practice. ${ }^{15}$ It, thereby, adds another dimension to understand a patient's preferences to treatment that might not be directly extrapolated from physiologic or clinical endpoints. ${ }^{36}$ When taken into the policy area, MID estimates could provide evidence regarding whether a treatment is beneficial or harmful to patients and whether information could help health policy-makers to 
determine the healthcare resource allocation and reimbursement strategy. ${ }^{37}$ Moreover, our findings bridge a knowledge gap regarding the influence of sex and age on MID estimates. Despite knowing such diverse results in subjective health and HRQoL, ${ }^{38}$ MID reflects an innate difference in patient preferences on HRQoL and well-being.

We must realise that EQ-5D is a generic instrument used to measure common domains of health that are relevant to the general population. ${ }^{39}$ The benefit of the MID estimates of GPBMs is unlikely to vary across different contexts. However, when considering patients with specific diseases, the condition-specific instrument might be more sensitive because its domains are fully focused on that condition. Despite some methodological limitations, the MID estimates derived in this study appear meaningful. The majority of ES values ranged from 0.3 to 0.5 , corresponding to a small to medium ES according to Cohen's criterion. Nonetheless, in the future, condition-specific instruments, such as a diabetes quality-of-life instrument, might be used to accrue extra evidence on the estimation of MID values.

Another point we need to consider is the MID estimates that may vary when considering the long-term effects of the treatment or care of people with multimorbidity. In our study, we found that patients at various ages show varying MID estimates. The psychological improvement and deterioration may affect the HRQoL responses to interventions, regardless of the presence of physical symptoms. ${ }^{13}$ These changes in HRQoL cannot be directly derived using PROMs; however, MID could provide information from the perspective of patients' well-being in which treatment could bring long-term meaningful changes to their health and facilitate appropriate decision-making of doctors. In the future, we need to consider the long-term effect on the estimation of MID values for patients with multimorbidity. Moreover, there is no consensus as to the 'best' method for MID estimation for HRQoL measures. ${ }^{40}$ Other methods, including the anchor-based method, should be included in the studies and may yield MID estimates in a plausible range rather than as a single value. ${ }^{35}$

This study has some limitations. First, only patients using public outpatient were recruited, which might not inclusively represent the overall general population. Although most people in HK use public services to manage their multimorbidity, it would be worth including the patients in private settings for further analysis. Second, the collected information on long-term conditions were self-reported instead of derived from medical records; therefore, no clinical information, such as the severity of the diseases or the type of diabetes, was included for the analysis that may possibly raise concerns regarding the validity of our findings. In addition, the proportion of younger patients in our sample was lower than the reference population, and this may limit the generalisability of our findings. Finally, the reliability of MID values estimated by only using instrumentbased method was limited, and different methods, for example, anchor-based and distribution-based methods, should be adopted in the future studies to examine the validity of our findings.
CONCLUSION

Our study presented four types of MID estimates of HK EQ-5D-5L value scores for patients with hypertension, diabetes and both conditions. We found that larger MID estimates were associated with higher utility scores, and the MID estimates for health improvement were larger than those of health deterioration. The MID estimates of the EQ-5D-5L should be further explored in different HK patient groups using different statistical methods.

Contributors ELW and RHX conceived and designed the study, RHX and AWC analysed the data. RHX prepared the manuscript. All authors read and approved the final manuscript.

Funding The authors have not declared a specific grant for this research from any funding agency in the public, commercial or not-for-profit sectors.

Competing interests None declared.

Patient and public involvement Patients and/or the public were not involved in the design, or conduct, or reporting, or dissemination plans of this research.

Patient consent for publication Not required.

Ethics approval This was a secondary data analysis, the ethical approval of the original patient experience survey was obtained from the Joint Chinese University of HK and the New Territory East Cluster Clinical Research Ethics Committee (Ref no.: CRE-2013.544).

Provenance and peer review Not commissioned; externally peer reviewed.

Data availability statement Data are available upon reasonable request. Data may be accessed by contacting with correspondence authors.

Open access This is an open access article distributed in accordance with the Creative Commons Attribution Non Commercial (CC BY-NC 4.0) license, which permits others to distribute, remix, adapt, build upon this work non-commercially, and license their derivative works on different terms, provided the original work is properly cited, appropriate credit is given, any changes made indicated, and the use is non-commercial. See: http://creativecommons.org/licenses/by-nc/4.0/.

ORCID iDs

Richard Huan XU http://orcid.org/0000-0002-4720-5172

Eliza Lai-yi WONG http://orcid.org/0000-0001-9983-6219

\section{REFERENCES}

1 de Boer IH, Bangalore S, Benetos A, et al. Diabetes and hypertension: a position statement by the American diabetes association. Diabetes Care 2017;40:1273-84.

2 Sowers JR, Epstein M, Frohlich ED. Diabetes, hypertension, and cardiovascular disease: an update. Hypertension 2001;37:1053-9.

3 Petrie JR, Guzik TJ, Touyz RM. Diabetes, hypertension, and cardiovascular disease: clinical insights and vascular mechanisms. Can J Cardiol 2018;34:575-84.

4 Ogurtsova K, da Rocha Fernandes JD, Huang Y, et al. IDF diabetes atlas: global estimates for the prevalence of diabetes for 2015 and 2040. Diabetes Res Clin Pract 2017;128:40-50.

5 Mills KT, Bundy JD, Kelly TN, et al. Global disparities of hypertension prevalence and control: a systematic analysis of population-based studies from 90 countries. Circulation 2016;134:441-50.

6 Wong ELY, Xu RH, Cheung AWL. Health-Related quality of life among patients with hypertension: population-based survey using EQ-5D-5L in Hong Kong SAR, China. BMJ Open 2019;9:e032544.

7 Wang W, Lau Y, Chow A, et al. Health-Related quality of life and social support among Chinese patients with coronary heart disease in mainland China. Eur J Cardiovasc Nurs 2014;13:48-54.

$8 \mathrm{Xu} \mathrm{RH}$, Cheung AWL, Wong EL-Y. Examining the health-related quality of life using EQ-5D-5L in patients with four kinds of chronic diseases from specialist outpatient clinics in Hong Kong SAR, China. Patient Prefer Adherence 2017;11:1565-72.

9 Pati S, Swain S, Knottnerus JA, et al. Health related quality of life in multimorbidity: a primary-care based study from Odisha, India. Health Qual Life Outcomes 2019;17:116.

10 Brazier J. Measuring and valuing health benefits for economic evaluation. Second ed. Oxford: Oxford University Press, 2007. 
11 Thissen D, Liu Y, Magnus B, et al. Estimating minimally important difference (mid) in PROMIS pediatric measures using the scalejudgment method. Qual Life Res 2016;25:13-23.

12 McClure NS, Sayah FA, Xie F, et al. Instrument-Defined estimates of the minimally important difference for EQ-5D-5L index scores. Value Health 2017;20:644-50.

13 Jayadevappa R, Cook R, Chhatre S. Minimal important difference to infer changes in health-related quality of life-a systematic review. $J$ Clin Epidemiol 2017;89:188-98

14 McClure NS, Sayah FA, Ohinmaa A, et al. Minimally important difference of the EQ-5D-5L index score in adults with type 2 diabetes. Value Health 2018;21:1090-7.

15 Crosby RD, Kolotkin RL, Williams GR. Defining clinically meaningful change in health-related quality of life. J Clin Epidemiol 2003:56:395-407.

16 Luo N, Johnson J, Coons SJ. Using instrument-defined health state transitions to estimate minimally important differences for four preference-based health-related quality of life instruments. Med Care 2010;48:365-71.

17 Ramos-Goñi JM, Oppe M, Slaap B, et al. Quality control process for EQ-5D-5L valuation studies. Value Health 2017;20:466-73.

18 Sullivan PW, Ghushchyan VH. EQ-5D scores for diabetes-related comorbidities. Value Health 2016;19:1002-8.

19 Rencz F, Gulácsi L, Drummond M, et al. EQ-5D in central and eastern Europe: 2000-2015. Qual Life Res 2016;25:2693-710.

20 Rowen D, Azzabi Zouraq I, Chevrou-Severac H, et al. International regulations and recommendations for utility data for health technology assessment. Pharmacoeconomics 2017;35:11-19.

$21 \mathrm{Xu} \mathrm{RH}$, Wong EL. Involvement in shared decision-making for patients in public specialist outpatient clinics in Hong Kong. Patient Prefer Adherence 2017;11:505-12.

22 Wong EL-Y, Cheung AW-L, Xu RH, et al. Development and validation of a generic patient experience instrument for measuring specialist outpatient service in Hong Kong. Int J Qual Health Care 2019;31:G158-64.

23 HerdmanM, GudexC Let al. Development and preliminary testing of the new five-level version of EQ-5D (EQ-5D-5L). An Int J Qual Life Asp Treat Care Rehabil - Off J Int Soc Qual Life Res 2011;20:1727-36.

24 RabinR O, Oppe M. EQ-5D-5L user guide. basic Inf how to use EQ-5D-5L Instrum, 2015. Available: http://www.euroqol.org/ fileadmin/user_upload/Documenten/PDF/Folders_Flyers/EQ-5D-5L_ UserGuide_2015.pdf

25 Wong ELY, Ramos-Goñi JM, Cheung AWL, et al. Assessing the use of a feedback module to model EQ-5D-5L health states values in Hong Kong. Patient 2018;11:235-47.

26 Wong EL-Y, Cheung AW-L, Wong AY-K, et al. Normative profile of health-related quality of life for Hong Kong general population using Preference-Based instrument EQ-5D-5L. Value Health 2019;22:916-24.

27 de Vet HCW, Terluin B, Knol DL, et al. Three ways to quantify uncertainty in individually applied "minimally important change" values. J Clin Epidemiol 2010;63:37-45.

28 Yost KJ, Eton DT, Garcia SF, et al. Minimally important differences were estimated for six patient-reported outcomes measurement information System-Cancer scales in advanced-stage cancer patients. J Clin Epidemiol 2011;64:507-16.

29 ClevelandR C. Terpenningl.STL: a Seasonal-Trend decomposition procedure based on Loess. J Off Stat 1990;6:3.

30 Wong ELY, Xu RH, Cheung AWL. Measuring the impact of chronic conditions and associated multimorbidity on health-related quality of life in the general population in Hong Kong SAR, China: a crosssectional study. PLoS One 2019;14:e0224970.

31 Tsiplova K, Pullenayegum E, Cooke T, et al. EQ-5D-derived health utilities and minimally important differences for chronic health conditions: 2011 Commonwealth fund survey of sicker adults in Canada. Qual Life Res 2016;25:3009-16.

32 SakibMN S, St.JohnP, et al. The prevalence of multimorbidity and associations with lifestyle factors among middle-aged Canadians: an analysis of Canadian Longitudinal Study on Aging data.(Clinical report). BMC Public Health 2019;19.

33 PeakJ, Goranitisl Det al. Predicting health-related quality of life (EQ5D-5 L) and capability wellbeing (ICECAP-A) in the context of opiate dependence using routine clinical outcome measures: CORE-OM, LDQ and top. Health Qual Life Outcomes 2018;16:1-11.

34 Jaeschke R, Singer J, Guyatt GH. Measurement of health status. ascertaining the minimal clinically important difference. Control Clin Trials 1989;10:407-15.

35 Revicki D, Hays RD, Cella D, et al. Recommended methods for determining responsiveness and minimally important differences for patient-reported outcomes. J Clin Epidemiol 2008;61:102-9.

36 Chen H, Taichman DB, Doyle RL. Health-Related quality of life and patient-reported outcomes in pulmonary arterial hypertension. Proc Am Thorac Soc 2008;5:623-30.

37 MouelhiY, JouveE Cet al. How is the minimal clinically important difference established in health-related quality of life instruments? review of anchors and methods. Health Qual Life Outcomes 2020;18:117-36.

38 Kirchengast S, Haslinger B. Gender differences in health-related quality of life among healthy aged and old-aged Austrians: crosssectional analysis. Gend Med 2008;5:270-8.

39 Chuang L-H, Garratt A, Brealey S. Comparative responsiveness and minimal change of the knee quality of life 26-item (KQoL-26) questionnaire. Qual Life Res 2013;22:2461-75.

40 Janssen B, Szende A, Cabases J. Self-Reported population health: an international perspective based on EQ-5D, 2014. 